\title{
Application of Green Silver Nanoparticles Synthesized using Leaf Extract of Tridax procumbens for Preparation of Clinical Antimicrobial Bandages
}

\author{
Vitthal Chopade*, Diksha Kamble \\ Department of Pharmaceutical Quality Assurance, Modern College of Pharmacy, SPPU, Pune, Maharashtra, INDIA.
}

\begin{abstract}
Objectives: To green synthesize silver nanoparticles by using weed plant Tridax procumbens also prepare and check antimicrobial activity of silver nanoparticles impregnated bandages against one gram positive bacteria and one-gram negative. Methods: The green synthesis of silver nanoparticles was done by chemical precipitation method using Tridax leaves extract as reducing agent. Synthesis of silver nanoparticles are characterized by UV-Visible spectroscopy, Dynamic light scattering (DLS), Zeta potential analysis, Fourier transform infrared spectroscopy (FTIR), Scanning Electron Microscopy (SEM) and X-ray diffraction analysis (XRD) to confirm size distribution, composition and crystalline nature of silver nanoparticles. Results: The peak was shown at $446 \mathrm{~nm}$ by UV-Vis spectrophotometer and Scanning Electron Microscopy analysis reveals the spherical -shaped nanoparticles with a mean size of $99 \mathrm{~nm}$. Fourier transform infrared spectroscopy proved that the phytochemicals in extract were responsible for formation of the silver nanoparticles and acted as reducing and stabilizing agent. XRD study have revealed the highly crystalline nature of nanoparticles. These nanoparticles were impregnated onto bandages and
\end{abstract}

they showed appreciable inhibitory activity against Escherichia coli $(6 \mathrm{~mm})$ and Staphylococcus aureus $(2 \mathrm{~mm})$. Conclusion: The characterization results shown the characteristic nature of the green synthesized silver nanoparticles. Thus the green synthesis was ecofriendly and nanoparticles showed promising antimicrobial activity. These antibacterial bandages can potentially be used for treating and open and infection-prone wounds. Key words: Tridax procumbens, AgNP's, Green synthesis of nanoparticles, SEM, Antimicrobial bandages, XRD.

\section{Correspondence}

Dr. Vitthal Chopade

Department of Pharmaceutical Quality Assurance, Modern College of Pharmacy, SPPU, Maharashtra, Pune, INDIA.

Phone: +919545452767

Email: vitthalchopade@gmail.com

DOI: 10.5530/ijpi.2021.1.3

\section{INTRODUCTION}

Nanotechnology is an important field of modern research, it deals with synthesis and manipulation of particle structure ranging from 1 to $100 \mathrm{~nm}$ in size. The small sized nanoparticles as compared to bulk material have different and enhanced properties and because of their small size they have a large surface area. ${ }^{1}$ Nanoparticles are synthesized by chemical and physical methods, these methods are quite expensive and hazardous to the environment. These methods involve use of toxic chemicals which are responsible for biological risks. The chemical synthesis approach have drawback that during the synthesis process, colloidal solution gets contaminated by several by-products due to chemical reaction. So to overcome these, development of green process for synthesizing nanoparticles is very necessary. The advancement of green synthesis over chemical and physical methods is ecofreindly, cost effective and easily scaled up for a large scale, which will not require high temperature, pressure, energy and toxic chemicals. Among all metal nanoparticles, silver nanoparticles have gained interest because of their chemical stability, good conductivity, catalytic, antimicrobial, anti-viral, antifungal properties. In biomedical applications, silver nanoparticles can be added to wound dressings, topical creams and antiseptic sprays, which acts as antiseptic and have a broad biocidal effect against microorganisms. To prevent growth of pathogens on the wound, a bandage impregnated with betadine or alcohol is commonly used. But they can cause allergic reaction to some people and delay the repair of wound. Tridax procumbens is a cosmopolitan weed. It grows in all types all soils, found everywhere and almost seen in all seasons. This plant has been used as wound healer in traditional medicine, so because of all this reasons, this plant is chosen for present investigation. Thus attempt has been made to green synthesize silver nanoparticles from Tridax procumbens, which reduces the silver ions present in the solution of silver nitrate. Also its antimicrobial activity is shown by preparing bandages impregnated with prepared green silver nanoparticles. ${ }^{2}$

\section{MATERIALS AND METHODS}

\section{Collection of plant materials and preparation of extract}

Tridax procumbens leaves were collected from Phaltan, Satara and authenticated. The leaves were collected, separated and washed with tap water following distilled water, then air dried at room temperature for 10 days and powdered further. $10 \mathrm{~g}$ of dried powder was mixed with $100 \mathrm{ml}$ of deionzed water and boiled on magnetic stirrer for $30 \mathrm{~min}$. The extract was allowed to cool and was filtered using Whatman no. 1 filter paper. The resulting filtrate was collected and used for further studies.

\section{Phytochemical investigation}

Qualitative phytochemical analysis of T. procumbens was performed using standard protocols described for determination of presence of several phytoconstituents like flavonoids, alkaloids, tannins, resins, saponins, steroids, carbohydrates, proteins, phenols, anthocyanins compounds. ${ }^{3}$

\section{Green synthesis of silver nanoparticles}

For green synthesis of silver nanoparticles, $10 \mathrm{ml}$ of leaf extract was added to $50 \mathrm{ml}$ of $1 \mathrm{mM}$ aqueous silver nitrate solution. The mixture was 
subjected to microwave irradiation for $5 \mathrm{~min}$. The visual color change in the reaction mixture from green to brownish black was observed which indicates the formation of silver nanoparticles. The solution was then centrifuged at 4,000 rpm for $25 \mathrm{~min}$. Obtained residue were silver nanoparticles, they were confined and concentrated by repeated centrifugation of mixture at 4,000 rpm for $25 \mathrm{~min}$. Each time, the supernatant was replaced with deionized water, so the particles are washed away which were not the capping agents. The suspension was dried and stored as crystalline powder for further studies. ${ }^{4}$

\section{Characterization of synthesized silver nanoparticles}

Ultraviolet-visible spectra analysis (UV-Vis)

The reduction of pure silver ions was examined by measuring surface plasmon resonance (SPR) of the reaction mixture by UV-Vis spectrum. $\mathrm{UV}-\mathrm{Vis}$ analysis scanning was done after reaction, time ranging from $5 \mathrm{~min}$ to $24 \mathrm{~h} .1 \mathrm{mg}$ sample was diluted with $5 \mathrm{ml}$ of deionized water and sonicated further for $60 \mathrm{sec}$. The solution was then analyzed by UV-visible spectroscopy in the range of 300-800 $\mathrm{nm}$ using UV-1800 Shimadzu, Japan. ${ }^{5}$

\section{Dynamic light scattering analysis (DLS)}

Nanoparticles were analyzed to determine the range of particle size using Horiba Nano Analyzer instrument. The size of the particles was measured by the time dependent fluctuation scattering of laser light when particles undergo Brownian movement. By this method mean particle size and size distribution pattern can be examined inside the sample.1mg of sample was diluted with $6 \mathrm{ml}$ of deionized water, sonicated for $60 \mathrm{secs}$ and was transferred into cuvette i.e. sample holders of the instrument and data was recorded. ${ }^{6}$

\section{Fourier transform infra-red spectroscopy analysis (FTIR)}

The FTIR of dried nanoparticles was studied using instrument Jasco, FT/IR-4100, Japan. 1mg of dried sample was mixed with $100 \mathrm{~g} \mathrm{KBr}$ powder and pellets were formed of $3 \mathrm{~mm}$ diameter. This pellets were further scanned in the range of $650-4000 \mathrm{~cm}^{-1}$ and resolution was kept as $4 \mathrm{~cm}^{-1}$.

\section{Scanning electron microscopic analysis (SEM)}

Scanning Electron Microscopy (SEM) analysis was done by using instrument JEOL JSM 6360A, INDIA. 20kv voltage was used for imaging nanoparticles. Thin films of the sample were prepared on a carbon coated copper grid by just dropping a very small amount of sample on the grid, extra solution was removed by blotting paper and then the film of SEM grid was allowed to dry under mercury lamp for $5 \mathrm{~min}$. As a result photograph is generated from insertion of the electron transmitted through the sample. ${ }^{8}$

\section{$X$ - ray diffraction analysis $(X R D)$}

The dried sample of silver nanoparticles were placed in the cavity of sample holder and was studied by Bruker D8 advance, INDIA with $\mathrm{Cu}$ $\mathrm{Ka}(\mathrm{l}=1.54 \AA)$ radiation, scanning range from 10E-90E. ${ }^{9}$

\section{Antimicrobial activity study of green synthesized AgNP's impregnated bandages.}

Two standard bacterial strains of gram negative bacteria Escherichia coli (NCIM No. 2065) (ATCC No. 8739) and gram positive bacteria Staphyllococcus aureus (NCIM No. 2079) (ATCC No. 6538P) were selected for evaluation. The antimicrobial activity was done by Agar Diffusion method. Alcohol was used as standard. Fresh overnight cultures of inoculum of each culture were spread on agar plates. Autoclaved sterile guaze bandages were cut to $1 \times 2 \mathrm{~cm}$ size and were impregnated with solution containing $30 \mu \mathrm{g} / \mathrm{ml}$ silver nanoparticles. Bandages immersed with nanoparticles were dried within the glass vial at $60^{\circ} \mathrm{C}$ in hot air oven. These bandages were then placed in the centre of agar plates and incubated at $37^{\circ} \mathrm{C}$ for $24 \mathrm{~h}$. The zone of inhibition was measured thereafter. ${ }^{10}$

\section{RESULTS}

\section{Phytochemical screening}

To evaluate various phytoconstituents in the leaf extract of Tridax procumbens, a series of qualitative photochemical tests were performed. The results are shown in Table 1 . The results of these are shown qualitatively as positive (+) or negative (-).

\section{Green Synthesis and characterization of silver nanoparticles}

Green synthesis of silver nanoparticles was accomplished by using ecofriendly and simple method using the leaves extract of $T$. procumbens. Microwave irradiation was used to accelerate the process of reaction. The formation of homogeneous silver nanoparticles was best achieved by an exposure of solution to microwave heating for $5 \mathrm{~min}$, result shown in Figure 1.

\section{Ultraviolet-visible spectra analysis (UV-Vis)}

UV-Vis spectroscopy analysis is used to determine the formation and stability of silver nanoparticles in aqueous solution. Surface Plasmon peak (SPR) wavelength can be tuned from $400 \mathrm{~nm}-530 \mathrm{~nm}$. In this present study, a single peak at $446 \mathrm{~nm}$ was observed. Broadening of the peak indicated that the nanoparticles are polydispersed. Hence, we can
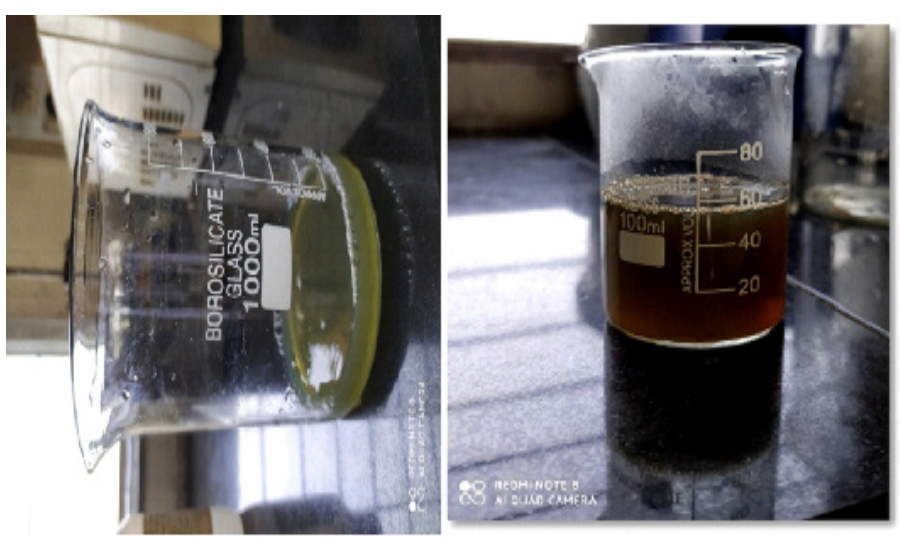

Figure 1: a) Mixture of AgNO3 solution and leaf extract. b) Synthesis of AgNP's and colour change of mixture after microwave irradiation.

Table 1: Phytochemical screening of aqueous leaf extract of Tridax procumbens.

\begin{tabular}{cc}
\hline Phytochemical Tests & Aqueous extract of T. procumbens \\
\hline Alkaloids & + \\
Flavonoids & ++ \\
Tannins & ++ \\
Saponins & + \\
Proteins & + \\
Phenols & + \\
Steroids & ++ \\
Anthocyanins & - \\
Cardiac glycosides & - \\
\hline
\end{tabular}


say that to synthesize silver nanoparticles, low concentration of silver nitrate is adequate. And nanoparticles formation is confirmed.

\section{Dynamic light scattering analysis (DLS)}

This analysis was done to examine particularly the measurement of monodispersity and size distribution in the aqueous solution. The $\mathrm{Z}$-average diameter of $143.2 \mathrm{~nm}$ with polydispersity index (0.306) was noticed in the size distribution graph, for green synthesized nanoparticles.

\section{Fourier transform infrared spectroscopy (FTIR)}

FTIR Spectrum shows the feasible phytoconstituents present in leaf extract which may be associated in the reduction of the silver ions. In our results, different absorption peaks were obtained. The strong absorption peaks at 1395 and $1652 \mathrm{~cm}^{-1}$ represents the presence of $\mathrm{NO}_{2}$ which might be from $\mathrm{AgNO}_{3}$ solution, the metal predecessor involved in silver nanoparticles synthesis process. Appearance of peak at 3501 $\mathrm{cm}^{-1}$ and $3646 \mathrm{~cm}^{-1}$ indicates the existence of phenolic hydroxyl $(\mathrm{OH})$, hydrogen bonds belonging to flavonoids and tannins. The peak at $1652 \mathrm{~cm}^{-1}$ represent carbonyl groups $(\mathrm{C}=\mathrm{O})$ from polyphenols such as catechin, epicatechin. The peak at $734 \mathrm{~cm}^{-1}$ represents the aromatic ring $\mathrm{C}-\mathrm{H}$ vibrations, indicates involvement of free catechin. The results imply that the flavonoids, tannins, phenolic compounds existing in the plant extract have been played major role in reduction of silver ions.

\section{Scanning electron microscopy (SEM)}

The silver nanoparticles are apparently seen at 6000x magnification in the photomicrograph. The particles are accumulated and were spherical in shape but accumulation has lead to elongated shape, but not welldefined. The size of nanoparticles were ranging from 42-159 nm and average size was $99 \mathrm{~nm}$, this was calculated using Image J software, result shown in Figure 2.

\section{X-ray diffraction analysis (XRD)}

XRD study established the presence of silver nanoparticles. XRD is mostly used for the phase identification and depiction of crystalline nature of synthesized silver nanoparticles. The Bragg reflections at $2 \mathrm{~h}=32.04^{\circ}$, $38.08^{\circ}, 45.94^{\circ}, 64.22^{\circ}, 77.38^{\circ}$ can be indexed to the (101), (111), (200), (220), (311) orientations, respectively, confirmed the presence of silver nanoparticles, result shown in Figure 2(d). Sharp peaks of XRD profile
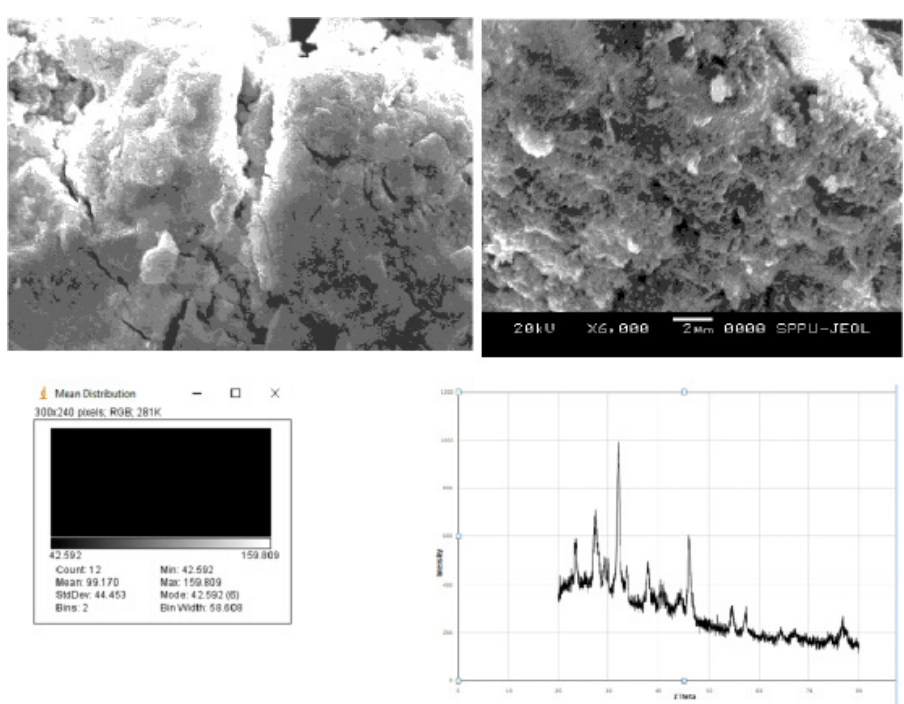

Figure 2: SEM images $(a, b)(c)$ Mean distribution of silver nanoparticles (d) XRD Pattern of AgNP's. clearly designate the highly crystalline nature of the synthesized silver nanoparticles. The XRD shows that silver nanoparticles developed are crystalline. X-ray diffraction spectra of pure crystalline silver structures have been issued by the Joint Committee on Powder Diffraction Standards (File No. 04-0738).

\section{Antimicrobial activity of AgNP's impregnated bandages}

In present study, the AgNP's impregnated bandages showed promising antimicrobial activity, result shown in Figure 3. After incubation, measured zone of inhibition for both the bacteria is shown in Table 2 .

\section{DISCUSSION}

The aqueous extract consists high quantity of flavonoids, tannins, phenolic compounds, ${ }^{11}$ which suggest that these phytoconstituents are major components for synthesis of these silver nanoparticles. The mechanism of green synthesis of silver nanoparticles is that $\mathrm{Ag}^{+}$have the potential to form intermediate composite with $-\mathrm{OH}$ or $\mathrm{C}=\mathrm{O}$ (Hydroxyl or carboxyl groups) present in the leaf extract, which comprises the reduction to $-\mathrm{COOH}$ forms and hence brings on bioreduction of $\mathrm{Ag}^{+}$to Ag nanoparticles. Color change of the solution is the initial confirmation of formation of silver nanoparticles. ${ }^{12}$ The leaf extract added to silver nitrate solution performed reducing activity as it contains bioactive agents and this resulted in color change of solution from greenish to brown on subjecting to microwave irradiation, indicating the formation of silver nanoparticles. The color change is due to Surface Plasmon vibrations in silver nanoparticles. It was confirmed by the UV spectroscopy. The particle size showed in DLS is bit large than the size reported by SEM analysis because of the variation in process of sample preparation and the DLS analysis measured particle size also includes the plant phytoconstituents covering the exterior of nanoparticles. It has been noted that the value of polydispersity index (PI) is less than 0.7 , which validates the quality of the nanoparticles to be fine. In FTIR, Studies have confirmed the fact that carbonyl group form amino acid

\section{Table 2: Zone of inhibition for gram positive bacteria and gram negative bacteria.}

\begin{tabular}{cc}
\hline Name of organisms & Zone of inhibition $(\mathrm{mm})$ \\
\hline & Sample of silver nanoparticles $(30 \mu \mathrm{g} / \mathrm{ml})$ \\
E.coli & $\mathbf{6}$ \\
S. aureus & 2 \\
\hline
\end{tabular}
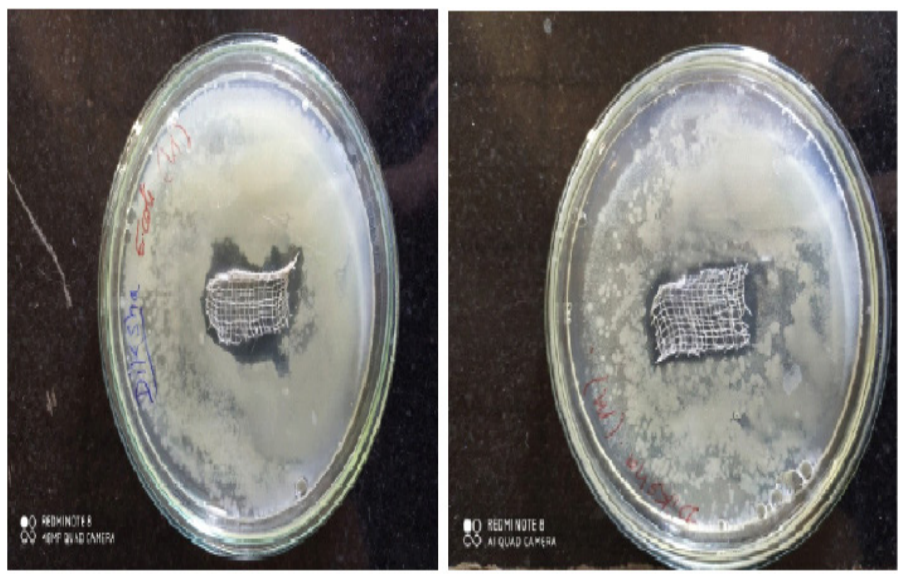

Figure 3: Antimicrobial activity of silver nanoparticles impregnated cotton bandages a) E. coli b) S. aureus. 
residues and protein have strong capacity to bind with metal. So the proteins could have formed layer over metal nanoparticles to prevent agglomeration. Shape, size and crystalline nature has been confirmed by SEM and XRD respectively. ${ }^{13}$ The AgNP's accumulate inside the bacterium and endeavor their effect by hampering enzymatic respiratory system of microbes, modification of DNA replication and interaction with S-H bonds of proteins directing towards inactivation and apoptosis of bacterial cell. However the antimicrobial activity by silver nanoparticles is well-known, but their mode of action is still debatable. ${ }^{14}$

\section{CONCLUSION}

Wound healing bandage is used commonly in surgical method. So the bandage was laced with nanoproduct in order to ease and fasten the healing process. Very purpose of preparing nanoparticles is to ensure effectiveness, efficacy and accuracy of the drug. Silver has very low systemic toxicity in higher animals like humans. Lowest concentration of nanoparticles was taken as it is effective enough. Silver ions is our drug in present study, it just accumulates in bacteria and kills it. The present study concluded that the plant Tridax procumbens can be used as an excellent source for green synthesizing the silver nanoparticles. The main advantage of green synthesis is that it is ecofriendly, inexpensive, without any need of toxic chemical reducing and capping agents. To prevent growth of pathogens on wound, a bandage infused with alcohol or betadine is used commonly. But this may give rise to allergic reaction in some patient and retard the repair of wound, so this AgNP's impregnated bandage can be used preferably. The antimicrobial activity of this bandage is also very promising because nanoparticles have small size and large surface area which shows high efficacy. Green synthesis is today's need, so researchers should focus more on ecofriendly ways of synthesis.

\section{ACKNOWLEGEMENT}

The authors would like to thank Modern College of Pharmacy, Yamunanagar, Nigdi, Pune, Savitribai Phule Pune University, Pune for providing the required facilities for research work.

\section{CONFLICT OF INTEREST}

The authors declare no conflict of interest with this research.

\section{ABBREVIATIONS}

AgNP's: Silver nanoparticles; AgNO $_{3}$ : Silver Nitrate; E. coli: Escherichia coli; S. aureus: Staphylococcus aureus; XRD: X-ray Diffraction Analysis; SEM: Scanning Electron Microscopy; FTIR: Fourier Transform Infrared Spectroscopy; DLS: Dynamic Light Scattering Analysis; UV-Vis: Ultraviolet Visible Spectroscopy.

\section{REFERENCES}

1. Dhanalakshmi T, Rajendra S. Synthesis of silver nanoparticles using Tridax procumbens and its antimicrobial activity. Arch Appl Sci Res. 2012;4(3):1289-93.

2. Ahmed S. A review on plants extract mediated synthesis of silver nanoparticles for antimicrobial applications: A green expertise. J Adv Res. 2016;7(1):17-28.

3. Sawant RS, Godghate AG. Preliminary phytochemical analysis of leaves of Tridax procumbens linn. Int J Sci, Env. 2013;2(3):388-94.

4. Sangeetha R, Dhanalakshmi N. Characterization of Silver Nanoparticles Synthesized Using The Extract of the Leaves of Tridax procumbens. Res J Med Plants. 2016;10(2):159-66.

5. Sundarranjan M, Jeelani A. Morphology studies on silver nanoparticles synthesized by green method using Tridax procumbens and Ocimum tenuiflorium leaf extracts. IJIRAE. 2017;4(9):50-4.

6. Khandel P, Shahi S, Soni D. Alpinia calcarata: Potential source for the fabrication of bioactive silver nanoparticles. Nano Convergence. 2018;5(37):1-17.

7. Khatami M, Varma R. Applications of green synthesized Ag, ZnO and Ag/ $\mathrm{ZnO}$ nanoparticles for making clinical antimicrobial wound-healing bandages. Sustainable Chemistry and Pharmacy. 2018;10:9-15.

8. Shital BK, Gaikwad DK. Integrated Management of linseed blight by green silver nanoparticles synthesized from fruit and leaves extract of morinda citrifolia L. Indo Global J Pharmaceutical Sciences. 2019;9(2):73-6.

9. Barai AC, Paul K, Dey A. Green synthesis of nerium oleander-conjugated gold nanoparticles and study of its in vitro anticancer activity on MCF-7 cell lines and catalytic activity. Nano Convergence. 2018;5(10):1-9.

10. Ondari NE, Nalini PM. Antimicrobial activity of biogenic silver nanoparticles synthesized using Tridax procumbens L. Int J Curr Res Aca Rev. 2014;2(7):3240.

11. Makarov W. "Green" Nanotechnologies: Synthesis of Metal Nanoparticles using Plants. Acta Naturae. 2014;6(1):35-44.

12. Anil S, Harish KS, Parvesh G. A Review on Tridax procumbens. Imperial J Interdisciplinary Res. 2016;2(8):308-19.

13. Pratik RC, Shalaka A, Suresh P. Effect of Biosynthesized Silver Nanoparticles on Staphylococcus aureus Biofilm Quenching and Prevention of Biofilm Formation. Nano-Micro Lett. 2012;4(1):34-9.

14. Pradheesh G, Suresh S, Suresh J. Antimicrobial and Anticancer Activity Studies on Green Synthesized Silver Oxide Nanoparticles from the Medicinal Plant Cyathea nilgiriensis Holttum. Int J Pharm Investigation. 2020;10(2):146-50.

Article History: Submission Date : 29-09-2020; Revised Date : 11-10-2020; Acceptance Date : 01-01-2021.

Cite this article: Chopade VV, Kamble DS. Application of Green Silver Nanoparticles Synthesized using Leaf Extract of Tridax procumbens for Preparation of Clinical Antimicrobial Bandages. Int. J. Pharm. Investigation. 2021;11(1):10-3. 